\title{
INCIDENCIA DE LAS TECNOLOGÍAS DE LA INFORMACIÓN Y LAS TELECOMUNICACIONES EN LOS HOTELES ESPAÑOLES
}

\author{
IMPACT OF INFORMATION AND COMMUNICATION \\ TECHNOLOGIES IN SPANISH HOTELS
}

\author{
Fernando J. Garrigós-Simón - PhD - garrigos@emp.uji.es \\ Daniel Palacios-Marqués - Ph.D \\ Yeamduan Narangajavana - Ph.D. Student \\ Universitat Jaume I \\ Department of Business Administration \\ 12080 Castellón de la Plana (Spain)
}

\begin{abstract}
RESUMEN
La introducción de las Tecnologías de Información y Comunicaciones ha supuesto un cambio de las reglas de juego para las empresas. Este impacto es especialmente importante en el sector turístico, por las propias peculiaridades del sector. Con el presente trabajo queremos investigar cómo el desarrollo de las nuevas tecnologías ha afectado el modo en el cual las empresas turísticas compiten y desarrollan sus tareas. A su vez, analizamos empíricamente el peso directo que tiene en los resultados empresariales el uso y desarrollo de las tecnologías de información y las comunicaciones. Para ello vamos a utilizar un cuestionario dirigido a directivos hoteleros y la metodología de ecuaciones estructurales. El estudio plantea los beneficios asociados al uso de las Tecnologías de Información y Comunicaciones y su impacto en la mejora de la acción empresarial.
\end{abstract}

Palabras Clave: Tecnologías de la Información y las Comunicaciones; Ecuaciones estructurales; Sector Hotelero

\section{INTRODUCCIÓN}

La incidencia de la industria de los servicios es clave en el desarrollo económico de todos los países. Su crecimiento deriva del desarrollo de la era de la información, que resalta el papel de los servicios en todo avance económico (RUST et al., 1996). La Tecnología ha sido un componente clave en el devenir de esta industria, especialmente, en las últimas décadas las tecnologías de comunicación e información (TIC) han afectado el modo en que las empresas de servicios compiten y desempeñan su tarea (COULTER, 1997,p.45; BUHALIS; LICATA, 2002). "los modos de hacer negocios y el entorno competitivo han cambiado", y en este marco la tecnología juega un rol fundamental en la competitividad de las organizaciones (BUHALIS, 1998; WAHAB; COOPER, 2001, p.320). 
Como un elemento clave dentro de la industria de servicios, el turismo se ha configurado como la industria más importante del mundo, tanto en términos de empleados, como por sus efectos en el desarrollo social y económico de las regiones o países (HOLJEVAC, 2003,p.130). El sector turístico no ha sido ajeno a los cambios en su entorno, estando por tanto inevitablemente asociados sus cambios a los desarrollos en nuevas tecnologías e innovaciones estructurales y organizativas (STAMBOULIS; SKAYANNIS, 2003, p.35). Más concretamente, la industria de viajes y turismo ha sido una de las pioneras en el desarrollo de las nuevas innovaciones, hecho que confiere a su comprensión un elemento imprescindibles en el actual marco de competencia en el sector.

En este trabajo vamos a analizar el impacto que tiene el desarrollo de las TIC en las empresas en la mejora de la competitividad, y por lo tanto en la explicación del desempeño de las empresas. Para atender a este objetivo este trabajo analiza en primer lugar la importancia de las TIC, y su papel crucial en la explicación del desempeño. A su vez, en la parte empírica se utiliza una muestra de 189 empresas hoteleras para corroborar las hipótesis teóricas.

\section{INFLUENCIA DE LAS TIC EN LA EMPRESA}

La 'Sociedad de la Información' ha sido caracterizada por : 1) la facilidad de acceso a la información, 2) la riqueza de interacciones, y 3) los bajos costes de información e interacción (KIM et al., 2002). Esta sociedad ha surgido fruto del desarrollo tanto de las tecnologías de información Y comunicación como de Internet. En este marco diferentes autores muestran cómo las TIC pueden ser un factor clave en el éxito de ciertas compañías. Enumeremos algunas de las ventajas del desarrollo de las TIC en las empresas turísticas.

Las nuevas tecnologías son esenciales para determinar la localización optima de los hoteles, porque sirven para observar el desarrollo de nuevos modos de transportes, como el desarrollo de grandes cruceros, trenes de alta velocidad, trenes y centros de embarque que conectan a los aeropuertos en las grandes ciudades, desarrollo de motores eléctricos o biológicos, concepción de zonas de la ciudad peatonales, para bicicletas o sin aparcamiento (PIZAM, 1999, HOLJEVAC, 2003). Además, las tecnologías de red permiten disponer de soluciones de movilidad para realizar cualquier actividad asociada al negocio, además de permitir la deslocalización de los recursos necesarios para la actividad empresarial (CONESA, 2003)

Las TIC y el comercio electrónico han "afectado rotundamente a las empresas en el modo en que ellas gestionan los proveedores, consumidores y las operaciones internas" (HANSON et al., 2002:4). Las TIC son clave en los procesos de reingenierías de negocios (DEVARAJ; KOLI, 2003), y esenciales para encarar la competición en el mercado global (CHEN; ZHU, 2004). De este modo, estas tecnologías proveen oportunidades significativas para reingeniería interna (sistemas de oficina, procesos administrativos, tratamiento de órdenes, sistemas de reserva, etc), y comercio electrónico (con empresas (B2B) o consumidores (B2c)) (HOLLAND et al., 1992; STAMBOULIS; SKAYANNIS, 2003, p. 37).

Las TIC son fundamentales para la automatización en operaciones rutinarias en los hoteles. De este modo, desde la reserva a la entrada y la salida, limpieza, auto-servicio,

Enc. Bibli: R. Eletr. Bibliotecon. Ci. Inf., Florianópolis, n. esp., 2º sem. 2008 
servicios de satisfacción a los consumidores, preparación de comidas, procesos de seguridad o control de empleados pueden ser simplificados y automatizados (PIZAM, 1999, HOLJEVAC, 2003). Los soportes electrónicos reducen la necesidad de espacio y simplifican el almacenamiento de información necesaria para el funcionamiento de las empresas. Además, la disponibilidad a bases de datos garantiza la accesibilidad a información, a la vez que asegura una actualización de datos adaptada a necesidades individuales (CONESA, 2003). A su vez, los hoteles pueden reducir costes, incrementar su poructividad (HOLLAND et al., 1992) y tasas de ocupación mediante el uso de las TICs, a través de tácticas de precios, subasta de últimas habitaciones o el calculo diario e incluso horario de los requerimientos de personal.

Las tecnologías de red permiten disponer de soluciones de movilidad para realizar cualquier actividad asociada al negocio. Igualmente permite la deslocalización de los recursos necesarios para la actividad empresarial (CONESA, 2003). De otro lado los hoteles podrían incrementar mediante el uso de las tecnologías de información su productividad y su tasa de ocupación. A través de acciones como la subasta de las últimas habitaciones, o el calculo diario e incluso horario de los requerimientos de personal.

Según Dussauge et al (1994) la tecnología es un factor que afecta a muchos aspectos de la estrategia de la empresa. Porter señaló que las TIC ofrecen nuevos caminos innovadores para competir a través de la reducción de costes y la diferenciación de productos. Otros, como Booth y Philip (1997) estudian la fusión entre una estrategia basada en las tecnologías de información para mejorar las eficiencias y crear ventajas estratégicas, y otra más dinámica y multidimensional centrada en la identificación las capacidades propias a través de consolidaciones tanto internas como externas (aprendizaje).

Las TICs son fundamentales para las conexiones entre diferentes áreas de los hoteles, mejorando las interconexiones en la cadena de valor, y reduciendo significativamente los costes de coordinación entre actividades y los riesgos inherentes a las transacciones (CLEMOS; ROW, 1991). Las innovaciones tecnológicas promueven la comunicación interna de las empresas, mejorando la comunicación entre divisiones o departamentos, constituyendo redes a parte de los flujos de comunicación y transformando el conjunto de la cadena logística (CONESA, 2003). Observese a este respecto la mejor integración entre la gestión del marketing, las reservas, las comidas y bebidas y las funciones del personal. Además sirven para simplificar tareas, mejorar la eficiencia y la calidad en el servicio, a la vez que racionalizar costes ${ }^{1}$.

Las TIC permiten en desarrollo de técnicas de gestión estratégica más moderna, y el acceso a sistemas de inteligencia expertos y consultores externos, que ayudan a mejorar la función directiva (REGNER, 2003, p.79). Nuevas tecnologías como los sistemas expertos incrementan la flexibilidad, la productividad organizacional, mejoran la consistencia en las tomas de decisiones, y fomentan oportunidades en los procesos de decisión (STOKES BERRY, 1998). Las tecnologías de la información son fundamentales para el apoyo a la gestión empresarial, mediante el desarrollo de la Inteligencia artificial que puede ayudar al desarrollo de nuevos productos hoteleros o

\footnotetext{
${ }^{1}$ Léase por ejemplo las TIC aplicadas a camareros para facilitar su conexión con la cocina en los restaurantes, aplicadas a clientes para la realización de tareas antes realizadas por el personal, o las conexiones entre las centrales de reserva, la recepción del hotel y departamentos variados como aprovisionamiento, cocina, etc...
}

Enc. Bibli: R. Eletr. Bibliotecon. Ci. Inf., Florianópolis, n. esp., 2º sem. 2008 
turísticos, o a la mejor realización de las funciones productivas. En este sentido, la literatura de dirección y administración de empresa ha remarcado las ganancias de eficiencia en aquellas industrias altamente integradas con redes ("networked") (Eng, 2004:87), o aquellas que han utilizado las nuevas tecnologías para mejorar las redes sociales o el establecimiento de comunidades virtuales (GARRIGÓS, 2008) ${ }^{2}$.

En este sentido, Un área potencialmente interesante para el uso de las tecnologías de información y comunicaciones es la gestión y el desarrollo de la creatividad y el aprendizaje organizativo (TIPPINS; SOHI, 2003). Los beneficios están asociados a conocer más y actuar antes que los competidores (LOEBBECKE; WAREHAM, 2003, p.178). A través del intercambio de información externa, los individuos podrían combinar y recombinar el conocimiento de dentro de la organización con nuevas ideas e innovaciones accesibles por individuos fuera de la organización, resultando en soluciones nuevas y creativas. En este sentido, Muchas organizaciones están implementando herramientas de comunicación basadas en Intranet, tales como redes electrónicas de discusión, para promocionar el intercambio de conocimiento a través de límites organizacionales internos (TEIGLAN; WASKO, 2003) Además, las nuevas tecnologías permiten disponer de forma sencilla y económica de informes y análisis elaborados por especialistas, inaccesibles o difícilmente disponibles de otra forma (CONESA, 2003). Dado ello, los directivos deberían primar acciones como la compra y la recolección de datos externos junto con tecnología de búsqueda estándar y novedosa (WALTERS et al., 2003).

A su vez, gracias a las tecnologías de información los hoteles pueden externalizar gran parte de sus funciones tradicionales tales como la contabilidad, seguridad, ingeniería, marketing, etc.. a empresas independientes, para reducir costes e incrementar la productividad (PIZAM, 1999, HOLJEVAC, 2003). Tal y como HJALAGER (2006) manifiesta, existe una carencia de visiones genuinas y detalladas sobre los cambios en la composición de las cadenas de valor, las cuales están recolocando activos económicos, empleo y ganancias en la industria más allá de las fronteras nacionales y regionales.

El gran poder de los turoperadores a nivel mundial ha producido signos de oligopolio y prácticas abusivas de comercio en los países de origen (SASTRE; BENITO, 2001; UJMA, 2001; BASTAKIS et al., 2004). Frente a ello, Buhalis (1998), ha argumentado que las TI e Internet pueden proveer herramientas estratégicas a las pequeñas y medianas empresas para desarrollar canales de distribución adicionales y alternativos, que les permitan comunicarse directamente con consumidores y distribuidores, aumentando su poder frente a estos. Las nuevas tecnologías permiten una mayor capacidad para acceder a mercados nuevos y más eficientes a las pymes, además de a una base más amplia de clientes potenciales, con el uso de conexiones electrónicas o la sustitución de las infraestructuras físicas por las tiendas virtuales (CONESA, 2003) ${ }^{3}$.

\footnotetext{
${ }^{2}$ Aunque el sector turístico se ha concentrado en la explotación de las TIC de una forma defensiva y limitada, como medios para reducir costes y acelerar el intercambio de la información y las transacciones (Stamboulis y Skayannis, 2003:35), autores como Quirchmayr y Schimak (1994) o el propio Garrigós (2008) muestran cómo sistemas multimedia, virtuales y la inteligencia artificial pueden mejorar los tradicionales sistemas de información en turismo.

3 Autores como Buhalis (1998)señalan las debilidades estratégicas e incapacidades tecnológicas de las pymes turísticas y regiones periféricas, proponiendo herramientas estratégicas para solventar estas deficiencias. También
}

Enc. Bibli: R. Eletr. Bibliotecon. Ci. Inf., Florianópolis, n. esp., 2º sem. 2008 
También es reslatable los cambios significativos ocurridos en la historia reciente en el marketing de alojamientos, debido al crecimiento de internet, con lo que las técnicas basadas en webs se han convertido en esenciales para el éxito de las ventas y el marketing en los hoteles (BREY et al., 2007:1408). Además, Buhalis (1998) manifiesta que la difusión de las interacciones basadas en TIC podrían resultar en la reducción de costes de transacción, llevando a procesos de desintermediación (STAMBOULIS; SKAYANNIS, 2003). Los centros de reserva mundiales permiten a los clientes finales reservar sus alojamientos preferidos en cualquier parte del mundo, siendo conducidas todas las ventas de forma electrónica y directamente con vendedores globales. Este hecho puede afectar al desarrollo de turoperadores, o a la transformación de las agencias de viaje en consultores turísticos.

Las nuevas tecnologías prometen muchas ventajas a las organizaciones, tales como la mejora en la eficiencia operativa, o la habilidad de proveer nuevos servicios para clientes (EDMONDSON et al., 2003, p.197), aumentando su valor añadido. Las TIC, a su vez, son esenciales en el nuevo escenario para "formular estrategias convenientes que al final soporten las relaciones con los clientes e incrementar y llevar la competitividad hacia nuevos niveles" (SEIDERS et al., 2000, p. 108). Por ejemplo, Chiou et al (2008) explican como la experiencia virtual proporciona actualmente a los consumidores la oportunidad de conseguir las sensaciones de sus viajes en Internet. Además, el mayor intercambio de información puede mejorar la calidad del producto y facilitar el desarrollo de nuevos productos (Eng, 2004:87) ${ }^{4}$. El uso de las TIC e Internet podrían ser importantes canales para ventas futuras en la industria hotelera (Dev y Olsen, 2000) $)^{5}$. No obstante es esencial no sólo su mero uso, sino también cómo utilizar estos medios. "En el futuro veremos lugares de valor añadido real, con personalización, recomendaciones de alternativas diferentes, asesores de viajes virtuales" (Watkins, 2000:40), y los directivos deberán saber instrumentar mecanismos apropiados para poder tratar de forma más eficiente con consumidores que tendrán más información sobre precios, calidades, etc., debido a la extensión de la red. "La transparencia de costes vuelve a los productos y servicios en 'commodities" (Sinha, 2000), lo cual transforma la forma de hacer negocios para poder obtener apropiados desempeños.

Atendiendo a Booth y Philip (1996), la importancia de las Tecnologías de Información reside en el papel que ellas juegan en las rutas de conducir a la competencia al éxito. De esta forma, autores como Bergeron et al (1991) mostraron que las compañías pueden usar el trabajo de Porter para identificar áreas donde las tecnologías de información pueden ser usadas en una moderna competencia ${ }^{6}$. Con la Nueva Economía, las empresas tienen que observar el alcance de las nuevas tecnologías para

\footnotetext{
destacan cómo los cambios tecnológicos han cambiado la concepción de los productos turísticos, ocasionando tanto amenazas como nuevas oportunidades a empresas y destinos.

4 Por ejemplo diversos lugares en Intenet abren algunos servicios a negociación. Por ejemplo, los hoteles los podrían usar para ofrecer a sus clientes productos suplementarios (como alquiler de coches, adquisición de viajes cortos o viajes de avión u otros transportes, tickets de teatro, etc.) a precios razonables.

${ }^{5}$ Es cierto que las reservas por Internet representaban en el año 2000 menos del cinco por ciento del total de reservas de los hoteles (Watkins, 2000:38), pero ellas han experimentado y tienen todavía un alto potencial de crecimiento, pudiendo a su vez complementar otros canales.

${ }^{6}$ No obstante, tampoco debemos utilizar las tecnologías de información exclusivamente para detectar los cambios del entorno. Los factores clave de la empresa son elementos fundamentales también a observar, como hemos comentado anteriormente
}

Enc. Bibli: R. Eletr. Bibliotecon. Ci. Inf., Florianópolis, n. esp., 2º sem. 2008 
saber discriminar precios y mejorar el poder frente a consumidores ${ }^{7}$, o saber como utilizar estas en las relaciones con consumidores u observar los requerimientos legales, términos y condiciones en los contratos de reserva de alojamiento vía páginas webs (Wilson, 2007). De acuerdo con Sinha (2000), el modo óptimo de contrarrestar la transparencia de costes es a través de la innovación, y "aquellos directivos que entiendan mejor las dinámicas de transparencias de costes en la red serán los más preparados para el cambio". Del mismo modo las tecnologías son también vitales para mejorar el poder frente a proveedores en el sentido de Porter. Con las nuevas tecnologías se puede acceder a una base de proveedores mucho mayor de la accesible mediante el comercio tradicional, además se eliminan fronteras, tanto en el aspecto de independencia física respecto al territorio o lugar de trabajo, como en lo relativo a la posibilidad de realizar transacciones comerciales de forma global con cualquier empresa (Conesa, 2003) ${ }^{8}$. Las TIC son vitales para superar ciertas asimetrías de información entre empresarios, a la ver de permitir la diferenciación informacional a las empresas ${ }^{9}$. Dadas todas estas asimetrías, según Fornell et al (1985), y Szeinbach et al., (1997), el ser pioneros en productos tecnológicos puede crear barreras de acceso a competidores, y costes de cambio para clientes. A través de ello también es posible para los proveedores establecer relaciones de lealtad con clientes que finalmente evaluarán la reputación de los proveedores para suministrarles productos de alta calidad.

De otro lado, las nuevas tecnologías de la información y comunicación aseguran contactos de negocios más rápidos y fáciles, sin la necesidad de viajes de negocios, léanse por ejemplo las conexiones vía Internet, video-conferencia o el desarrollo del correo electrónico ${ }^{10}$. Este hecho está afectando al diseño e introducción de las TIC en los hoteles, y también a la diversificación de los hoteles urbanos y de negocios hacia nuevas modalidades de ocio, con la posibilidad de separación y trato diferente a ambos segmentos gracias al uso de las TIC (Garrigós y Narangajavana, 2002, 2006). En este sentido, autores como Feiertag (2007) exponen cómo utilizar la tecnología por ejemplo para planificar reuniones en los hoteles.

\footnotetext{
${ }^{7}$ En esta situación, las organizaciones deben buscar en qué tipo de productos podrán discriminar precios, observando que las posibles asimetrías de información frente a clientes y el coste de los consumidores de búsqueda de información se reducirán. No obstante, esta reducción no será asimétrica en todos los productos y servicios, con lo que deberán saber los alcances de las nuevas tecnologías para poder averiguar cómo discriminar.

${ }^{8}$ Por ejemplo algunas páginas Web mostradas por autores como Seiders et al. (2000), o Sinha (2000), permiten a las compañías pertenecientes a un amplio rango de industrias encontrarse unas a otras, lo cual puede ser fundamental para obtener relaciones con proveedores apropiados, además de identificar productos, hechos y precios con una precisión mucho mayor. A ello se une el hecho de que los directivos pueden adquirir un mayor conocimiento sobre estructuras de costes, información gran parte de la cual anteriormente sólo era posible con procesos de integración vertical, y que ahora, debido a las innovaciones de Internet, pueden ser conocidas por los directivos y usadas por ellos para mejorar el poder y adquirir ventajas frente a proveedores tradicionales. Es el caso del llamado B2B ("business to business").

${ }^{9}$ Esta diferenciación resulta de la información desigual del consumidor acerca de características (existencia, precio, calidad, etc.) de varios productos. Casos de diferenciación serían a su vez los llamados "bienes experiencia". Desde la Teoría de la Agencia, Sharma (1997) señala también las consecuencias de sustanciales asimetrías de conocimiento que favorecen a agentes profesionales en sus relaciones de mercado.

${ }^{10}$ No está demostrado que esto afecte negativamente al desarrollo global del sector turístico(Cooper et al. 1998). A ello se añade el hecho de que el número de personas que viajan por placer, ocio o aprendizaje incrementará significativamente en el futuro(Holjevac, 2003).
}

Enc. Bibli: R. Eletr. Bibliotecon. Ci. Inf., Florianópolis, n. esp., $2^{\text {o }}$ sem. 2008 
Las TIC juegan un papel significativo para ayudar a las organizaciones a percibir cambios en su entorno (Laudon y Laudon, 1998) ${ }^{11}$. La tecnología puede mejorar la comunicación interna y externa en las organizaciones. Ella es fundamental para integrar cómo una organización interpreta qué está ocurriendo ahí fuera con la codificación de "cómo hacemos las cosas aquî" (Barnatt, 1996:196). Las empresas necesitan integrar el nuevo conocimiento que encuentran en el entorno externo para permanecer competitivas (Teigland y Wasko, 2003:268). La investigación en TIC permite la creación y difusión del conocimiento desde y a través de fuentes dispersas y globales. Actualmente, las redes de ordenadores sobrepasan límites físicos, espaciales y temporales para conectar gente y crear redes de ocio. Estas conexiones fortalecen relaciones sociales, permiten la comunicación de ideas para crear consenso entre un mayor número de gente, y promueve la cooperación y compartimiento del conocimiento entre los diferentes departamentos y oficinas en una empresa, abiendo las oportunidades para colaborar e incrementando las posibilidades y la riqueza de los recursos de la empresa (Sambamurthy et al, 2003). Estas tecnologías permiten el aprendizaje sobre las oportunidades derivadas de la colaboración y comportamiento mutuo del conocimiento, y producen una retroalimentación y la explotación de sinergias en toda la empresa (Broadbent et al., 1999; Weill y Ross, 2005). Además, ellas también promueven las relaciones entre organizaciones (Youndt et al., 2004: 342) o individuos a través de la empresa .Sin embargo, la habilidad de las empresas para asimilare información externa y nueva es en gran parte una función de la habilidad de las empresas para procesar internamente la información (Teigland y Wasko, 2003:270), y aquí el desarrollo de las TIC juega un papel vital. La comunicación interna y externa de la empresa se facilita, favoreciendo el desarrollo de nuevas estrategias de colaboración con el entorno (Conesa, 2003). En casi todos los países los alojamientos pequeños, independientes y flexibles dominan el mercado, y juegan un role vital no solo en términos estructurales, sino también en términos de su contribución al producto nacional bruto y al empleo ${ }^{12}$. Tal y como Altinay y Wang (2006:430) remarcan " la naturaleza altamente competitiva y volátil del entorno global de hoy en día, motiva a las organizaciones a buscar colaboración con otras empresas". En este sentido, frente a los procesos de concentración que poniendo en peligro la competitividad de las PYMEs, las TIC pueden y permiten el desarrollo de alianzas estratégicas y agrupaciones de hoteles independientes, que combinan los beneficios asociados a las economías de escala de las grandes cadenas hoteleras, con las ventajas de flexibilidad asociadas a las pequeñas empresas (Garrigós y Narangajavana, 2002, 2006). Atendiendo a Pfeffer (1992:88), jurisdicción sobre recursos (como es la información) es una fuente importante de poder. Sin el desarrollo de las tecnologías de información no podría explicarse la conformación y desarrollo de las alianzas estratégicas y de las

\footnotetext{
${ }^{11}$ Hoy en día, todo el mundo está bombardeado con datos, el problema es cómo almacenarlos y tenerlos disponibles en un momento dado. La esencia de la intuición descansa en la organización del conocimiento para una rápida identificación, y las TIC pueden ayudar a este proceso (Mintzberg et al., 1998).

${ }^{12}$ Por ejemplo, se estima que más del $90 \%$ de los hoteles europeos son pequeñas y medianas empresas gestionadas familiarmente (European Commission, 2002; Bastakis et al., 2004).
}

Enc. Bibli: R. Eletr. Bibliotecon. Ci. Inf., Florianópolis, n. esp., 2ºm. 2008 
redes interempresariales, aspecto que, como señalan muchos autores de la Teoría de la Agencia, favorece a su vez un incremento de disponibilidades de información ${ }^{13}$.

Las TIC son importantes para conocer el desarrollo de los competidores, nuevas formas de turismo, o posibles nuevos negocios, como pueden ser destinos turísticos bajo el agua, cruceros submarinos, resorts especializados en personas con discapacidades, parques temáticos o islas fantásticas, etc... . A su vez, las TIC son fundamentales para el diseño y desarrollo de los "hoteles verdes" o "ecohoteles", o para la preservación del entorno o la naturaleza (Pizam, 1999). Todos estos aspectos pueden afectar tanto la elección de la localización, como las formas de construcción, equipamiento, productos y servicios de los hoteles.

A pesar de la importancia de estas ventajas tecnológicas, ¿puede ser la situación de una compañía replicada por otra cuando los sistemas de información pueden ser tan rápidamente copiados? — se preguntan Booth y Philip (1997). Atendiendo a Chapelet y Tovstiga (1998), las empresas exitosas se concentran en mantener medidas para proteger sus capacidades intelectuales, así como suelen "escanear" su entorno constantemente en busca de nuevos recursos tecnológicos. Según Schoemaker (1997:59), la razón de un mayor rendimiento promedio de algunas empresas sólo deriva de activos y capacidades difíciles de imitar. Algunos investigadores manifiestan que los recursos físicos en TIC són faciles de imitar (Clemons y Row, 1991; Bruque et al., 2003, Wade et al, 2004), con lo que ellos difícilmente podrían generar ventajas competitivas (Mata et al., 1995). No obstante, estas críticas sólo serían válidas si atendemos a las tecnologías de información como meros recursos estratégicos y no tienen en cuenta las sinergias de los sistemas integrados (Bharadwaj, 2000). Así consideradas, Drury (1997) critica a su vez que mientras la tecnología está constantemente cambiando, las estructuras de tecnologías de información permanecen relativamente estables, manifestando su desacuerdo en que las empresas se fundamenten más en la ventaja tecnológica y en la competencia estratégica que en el control de los sistemas de información, sistemas que mejoran la toma de decisiones y la efectividad de las tecnologías de información. Tal y como O'Connor y Frew (2004:184) argumentan, "la evaluación en el contexto de sistemas basados en tecnologías de la información es compleja y multi-facética". Los trabajos empíricos iniciales no encontraron un efecto directo positivo entre las TIC y el desempeño (Mahmood y Mann, 1993). Sin embargo, autores tales como Powell y Dent-Micallef (1997), Bruque et al., (2003), Devaraj y Kohli (2003), Tanriverdi (2005) o Weiss y Ross (2005) han encontrado que las TICs pueden activar otros recursos intangibles. Ellos podrían incluir estrategias de negocio, diseño organizativo, estructuras, capacidades organizativas y humanas, competencias o cultura organizativa a favor de la innovación y el intercambio de la información, o el desarrollo del aprendizaje y prácticas de dirección del conocimiento en las organizaciones, como hemos explicado previamente, todas las cuales están relacionadas con la consecución de desempeño

\footnotetext{
13 Autores como Hamel (1991) analizan cómo pueden actuar las alianzas como transmisoras de innovación, información y conocimiento. Russ y Camp (1997) corroboran esta visión y desarrollan un modelo teórico que conecta las areas de alianzas estratégicas y la transferencia de tecnología. Ingram y Baum (1997:72) también enfatizan la importancia de la transferencia del conocimiento cuando los hoteles están afiliados a cadenas hoteleras, y Darr et al. (1995) concluyen que los restaurantes adscritos a cadenas aprenden más rápidamente al estar expuestos a la experiencia de la cadena.
}

Enc. Bibli: R. Eletr. Bibliotecon. Ci. Inf., Florianópolis, n. esp., 2º sem. 2008 
superior. Además, estudios recientes de Bharadwaj (2000) y Santhaman y Hartono (2003) defienden un impacto positivo y directo de las TICs en el desempeño de las empresas.

De acuerdo con lo anterior, podemos hipotetizar que las empresas con las TIC más desarrollaras obtendrán un desempeño mayor que aquellas que no poseen estos sistemas. Esta hipótesis están expresadas en la Figura 1, donde $\xi_{2}$ indica la variable latente que resume el desarrollo de las TICs en la empresa y $\eta_{1 \mathrm{i}}$ expresa la medida de los diferentes medidas de desempeño de la empresa

\section{METODOLOGÍA}

El objetivo de este trabajo es el contrastar las relaciones causales entre las TIC y el desempeño. La medición del desarrollo de las TIC en las empresas (escala de 4 ítems) y el desempeño empresarial (escala de 10 ítems basada en Camisón, 1999) fue realizada a partir de partir de un cuestionario enviado por correo electrónico a los directores generales de empresas hoteleras radicadas en toda España que proveen sus direcciones electrónicas en las principales páginas web especializadas de España. Excluyendo hostales y pensiones, la población final totalizó aproximadamente 3500 empresas hoteleras. La muestra final se compuso de 189 empresas, cuyo ratio de respuesta dio un error de muestra de $\pm 5,48 \%$ para un nivel de confianza del $95 \%$. La muestra incluyó hoteles de ciudad $(31,2 \%)$, de sol y playa $(21,2 \%)$, rurales $(39,7 \%)$ y balnearios $(7,9 \%)$. La mayoría de la muestra consistio en PYMEs (menos de 250 empleados), con un $44,4 \%$ de organizaciones clasificadas como micro empresas (menos de 10 empleados). El cuestionario recoge preguntas cerradas, utilizando escalas multi-item Likert de 7 puntos, con un rango de creciente importancia comparando con competidores como Slater y Olson (2000). La base de datos se creó utilizando el programa SPSS.

\subsection{Escalas utilizadas}

Para observar el desarrollo de las tecnologías de información en las empresas hemos desarrollado una escala de 4 ítems. Estos ítems recogen, en opinión de los directivos, el desarrollo o existencia en las empresas de: 1) Muchos y buenos recursos técnicos para la recogida y generación de información 2) Tecnología hardware (ordenadores, networks, Intranets...), 3) Herramientas software y de gestión de datos (bases y almacenes de datos, herramientas de gestión de documentos y de gestión del flujo de trabajo...), y 4) Herramientas inteligentes (inteligencia artificial para el apoyo de decisiones, realidad virtual, motores de búsqueda, mapas de conocimiento...). La medición del desempeño se basó en una escala reducida de 10 items obtenida de Camisón (1999). Ambas escalas utilizan medidas subjetivas. Tradicionalmente, los datos cualitativos e indicatores financieros han sido utilizados por ser visto como más rigurosos. Sin embargo, los datos cuantitativos pueden ser de limitado uso en cientos estudios (Pizam y Mansfeld, 1999:309). La operativización cualitativa seguida en este artículo es una práctica "típica" y bien aceptada en la investigación estratégica (Campbell-Hunt, 2000:146). Teóricamente, la investigación ha sugerido que las percepciones directivas son a veces más criticas del desempeño que indicadores objetivos que son "mentalmente distantes" (Chattopadhyay et al., 1999). Lyon et al 
(2000: 1058-1059) sugieren que la percepción directiva provee la mejor evaluación de las condiciones dentro de una empresa. La validez de esta medida es a su vez corroborada por diversos autores, que observan una fuerte asociación entre este tipo de medidas y las medidas objetivas ( Venkatraman y Ramanujam, 1986).

\subsection{Métodos de análisis}

En este trabajo utilizamos la metodología de las ecuaciones estructurales en dos pasos, tanto para validar las escalas como también para establecer las relaciones causales entre "el desarrollo de las tecnologías de información" y el desempeño (Anderson y Gerbing, 1988). Este recurso metodológico significó una innovación en la literatura desde que es capaz de representar conceptos latentes desde variables observadas y de estudiar las relaciones causales con datos no experimentales, cuando estas relaciones son de tipo leneal. Se utilizó el programa estadístico EQS 5.7b. Este programa utiliza el método de Máxima Verosimilitud por defecto, ofreciendo estimadores consistentes para muestras amplias, con variables continuas y distribuciones multi-normales (Bollen, 1989). Sin embargo, nuestra investigación viola la asunción de normalidad multivariante, dado que al utilizar escalas Likert no utiliza variables continuas. En esta situación, algunos autores como Satorra y Bentler (2001) sugieren la utilización de otros métodos tales como los Estimadores Robustos Estándar y los chi-cuadrado Sattorra-Bentler, que han sido utilizados en nuestro trabajo.

La aproximación en dos fases esencialmente comprende el análisis de medidas de las variables latentes, y el estudio de su causalidad en un modelo estructural. Siguiendo a Bollen (1989), procedimos al análisis de dimensionalidad, fiabilidad y validez de las escalas. Examinamos la dimensionalidad de la escala que mide el desarrollo de las TIC dentro de las empresas utilizando análisis factorial confirmatorio (AFC). Además, in el caso del desempeño y con el objeto de garantizar la convergencia de los resultados, desarrollamos previamente un análisis factorial exploratorio (AFE), cuya dimensionalidad sirvió para el posterior análisis confirmatorio. Para corroborar la conveniencia del mismo utilizamos la matriz de correlaciones, el contraste de esfericidad de Barlett, y la Medida de Adecuación Muestral, calculada de manera global con el estadístico de Kaiser-Meyer-Olkin y para cada variable. Para la estimación de los factores y valoración del ajuste general hemos escogido el análisis de componentes principales, apropiado para resumir la información original en factores con propósitos prospectivos. El número de factores se determinó en función del criterio de la raíz latente, que considera factores con autovalor o raíz latente elevado (normalmente superior a la unidad). Finalmente, para la interpretación de los factores utilizamos la rotación ortogonal mediante el método VARIMAX.

\section{PRINCIPALES RESULTADOS}

\subsection{Evaluación de las escalas de medida}

El análisis de dimensionalidad intenta probar la existencia de un concepto singular en todos los indicadores que forman una escala (Anderson y Gerbing, 1988). Para ello utilizamos la técnica del AFC, con objeto de contrastar el modelo expuesto en la Figura 1. En la Tabla 1 señalamos las medidas de bondad del ajuste del modelo indicativos de la dimensión individual de las TICs. La observación de todos y cada uno de los 
resultados corrobora la bondad del ajuste realizado. En primer lugar observamos que el modelo está sobreidentificado, con dos grados de libertad. En relación a las medidas absolutas de ajuste, el estadístico sobre la $\chi^{2}$ de Satorra-Bentler nos indica su elevada significatividad estadística, al ser esta mayor de 0,05. Además, el GFI se sitúa por encima de 0,9 y el RMSEA por debajo de 0,08 en todos los modelos, lo que nos indica valoraciones óptimas. Con relación a las medidas incrementales de ajuste, todas ellas (AGFI, BBNFI, BBNNFI, RCFI y IFI) superan el 0,9, como estipula la teoría. Finalmente, atendiendo a la medida de parsimonia, el NC tiene un valor menor a 1 que podrían ser indicativo incluso de un sobreajuste del modelos. Finalmente, todos los estimadores estandarizados de los parámetros son estadísticamente significativos para un nivel de confianza del $95 \%$. Además, podríamos constatar que en las cargas estadísticas son bastante elevadas.

Tabla 1. Medidas de bondad de ajuste del modelo que mide el desarrollo de las TI.

\begin{tabular}{|c|c|c|c|c|c|c|c|c|c|c|c|}
\hline & \multicolumn{7}{|c|}{ INDICES DE AJUSTE DE TIC } \\
\hline & $\begin{array}{c}\text { Satorra- } \\
\text { Bentler } \chi^{2}\end{array}$ & $\begin{array}{c}\text { Grados } \\
\text { de } \\
\text { libertad }\end{array}$ & $\begin{array}{c}\text { Nivel } \\
\text { de sign. }\end{array}$ & GFI & RMSEA & AGFI & BBNFI & BBNNFI & RCFI & IFI & NC \\
\hline $\begin{array}{l}\text { Medidas } \\
\text { de ajuste }\end{array}$ & 1.439 & 2 & 0,486 & 0,996 & 0,000 & 0,980 & 0,995 & 1,005 & 1 & 1,001 & 0,72 \\
\hline
\end{tabular}

La fiabilidad puede ser definida como el grada en el cual las medidas están libres de cualquier sesgo resultante de errores causales, y dan los mismos resultados independientemente del modelo (Hair et al., 1998). Siguiendo Bollen (1989), estimamos la fiabilidad individual de los indicadores con el coeficiente de correlación múltiple al cuadrado. Podemos observar que el índice del último indicador individual es menor que el valor mínimo, 0,5, sin embargo, la fiabilidad compuesta del constructo produce yna elevada fiabilidad, ya que es superior a 0,7, ratificando así la precisión de nuestro análisis.

El análisis de validez intenta o convergente pretende comprobar que lo que se está midiendo sea verdaderamente el constructo que se pretende valorar, o que los diversos ítems utilizados para medir el concepto están correlacionados fuerte y positivamente. El ajuste de los modelos, especialmente debido a la bondad de medidas de ajuste incremental como como el AGFI o la BBNFI corroboran esta validez. La validez discriminante indica el grado en el cual dos medidas desarrolladas constructos similares pero conceptualmente diferentes están relacionadas. Al conformar el desarrollo de las TICs como una única dimensión, no debemos realizar ningún análisis de validez discriminante.

Para dimensionalizar la escala de medida del desempeño, hemos optado por el uso de un AFE (tabla 2). El número de factores a extraer, de acuerdo con el criterio de raíz latente, sugiere una solución de 4 factores. En esta clasificación la medida de adecuación muestral de Kaiser-Meyer-Oklin alcanza un valor de 0,806, mientras que las medidas de adecuación muestral individuales sobrepasan en todos los casos el 0,7, excepto en un caso en el que es de 0,637 . Si atendemos al contraste de esfericidad de 
Barlett, nos indica un $\chi^{2}$ de 1177,188 (con 45 grados de libertad, $\mathrm{p}<0,000$ ). Los cuatro factores a extraer de acuerdo con el criterio de raíz latente indican autovalores de $4,615,1,695,1,077$ y 1,060 respectivamente. Además, esta alternativa nos explica el $84,460 \%$ de la varianza. Su interpretación implica la distinción entre la Rentabilidad Financiera (Rent., ítems 1, 2 y 3), los Resultados de Crecimiento (Crec., ítems 4, 5 y 6), Satisfacción de Stakeholders (S.Stak., ítems 7 y 8), y Posición Competitiva (P.Comp.,ítems 9 y 10).

Sin embargo, y en aras a una mayor rigurosidad, hemos establecido un AFC para la variable global desempeño (DESTOT). Para ello, hemos tratado a la variable desempeño como una variable latente de segundo orden inducida por las cuatro dimensiones sugeridas por el AFE, definidas como variables latentes de primer orden fomadas de indicadores que son las variables observadas. Para reducir la complejidad de los modelos estructurales y facilitar estimaciones posteriores (Landis et al., 2000) utilizamos una caracterización normal en la literatura utilizando variables compuestas (con la suma de los indicadores) para cada dimensión. La observación de todos los índices corrobora la bondad de nuestro ajuste (Tabla 3). En primer lugar, el modelo esta sobreidentificado con dos grados de libertad. La calidad de las medidas de ajuste absolutas, incrementales y de parsimonia de los modelos establicidos también se puede corroborar (Hair et al., 1998). El AFC nos permite validar la dimensionalización alcanzada previamente con el AFE. Se puede constatar además que en el modelo todos los parámetros son estadísticamente significativos para un nivel de confianza del $95 \%$, y las cargas factoriales son superiores a 0,4 , hecho que nos remarca la validez convergente de la escala. La fiabilidad del constructo también es aceptable, aunque ajustada $(0,67)$; además, la utilización del Alpha de Cronbach nos da un valor mayor a 0,69 .

Tabla 2. Desempeño organizativo. Análisis Factorial de Componentes Principales con solución de 4 factores.

\begin{tabular}{|c|c|c|c|c|c|}
\hline & $\begin{array}{c}\text { Factor } 1 . \\
\text { Rent. }\end{array}$ & $\begin{array}{c}\text { Factor } 2 . \\
\text { Crec. }\end{array}$ & $\begin{array}{c}\text { Factor } 3 . \\
\text { S.Stack }\end{array}$ & $\begin{array}{l}\text { Factor } 4 . \\
\text { S.comp.. }\end{array}$ & Comunalidad \\
\hline \multicolumn{6}{|l|}{ ITEM } \\
\hline R1=Rentabilidad económica media & 0,912 & 0,154 & 0,049 & 0,126 & 0,875 \\
\hline R2=Rentabilidad financiera media & 0,917 & 0,164 & 0,102 & 0,106 & 0,890 \\
\hline R3 =Rentabilidad media en ventas & 0,874 & 0,357 & 0,022 & 0,085 & 0,899 \\
\hline $\mathrm{R} 4=$ Crecimiento medio de ventas & 0,229 & $\mathbf{0 , 8 7 8}$ & 0,099 & 0,093 & 0,842 \\
\hline R5 = Ganancia de cuota de mercado & 0,207 & $\mathbf{0 , 8 7 3}$ & 0,157 & 0,176 & 0,861 \\
\hline R6=Creación de riqueza & 0,274 & 0,724 & 0,292 & 0,309 & 0,781 \\
\hline R7 =Satisfacción de los clientes & $-0,073$ & 0,264 & 0,896 & $-0,015$ & 0,877 \\
\hline R8 =Satisfacción de los empleados & 0,233 & 0,086 & $\mathbf{0 , 8 4 0}$ & 0,304 & 0,860 \\
\hline R9 =Posición competitiva global & 0,158 & 0,065 & 0,188 & $\mathbf{0 , 8 5 1}$ & 0,788 \\
\hline $\begin{array}{l}\text { R10 = Tasa de éxito en el lanzamiento de } \\
\text { nuevos productos }\end{array}$ & 0,066 & 0,329 & 0,032 & 0,812 & 0,773 \\
\hline Autovalores & 4,615 & 1,695 & 1,077 & 1,066 & \\
\hline$\%$ de varianza explicado & 46,147 & 16,950 & 10,767 & 10,596 & \\
\hline
\end{tabular}

Tabla 3. Modelo global de desempeño (Desempeño Total). Bondad de ajuste del AFC. 


\begin{tabular}{|c|c|c|c|c|c|c|c|c|c|c|c|}
\hline & \multicolumn{11}{|c|}{ INDICE DE AJUSTE DEL DESEMPEÑO } \\
\hline & $\begin{array}{l}\text { Satorra- } \\
\text { Bentler } \chi^{2}\end{array}$ & G.L. & $\begin{array}{l}\text { Nivel } \\
\text { sign. }\end{array}$ & GFI & RMSEA & AGFI & BBNFI & BBNNFI & RCFI & IFI & NC \\
\hline $\begin{array}{l}\text { Medidas de } \\
\text { ajuste }\end{array}$ & 3.02 & 2 & 0.220 & 0.991 & 0.065 & 0.953 & 0.975 & 0.966 & 0.988 & 0.989 & 1.51 \\
\hline
\end{tabular}

No obstante, nuestro objetivo es reducir todavía más el número de factores para poder atender al análisis de los modelos estructurales causales. Dado ello, y la observación de los dos últimos autovalores, muy cercanos a la unidad, optamos por la evaluación de un Análisis Factorial Exploratorio de dos factores. Su interpretación implica la distinción entre rentabilidad financiera, (ítems 1,2 y 3) y los otros 7 ítems, que indican la posición competitiva global de las empresas. En esta clasificación las medidas individuales de adecuación muestral y el estadístico Kaiser-Meyer-Oklin son similares a las obtenidas en el anterior modelo. Lo mismo sucede con el contraste de esfericidad de Barlett, que sigue indicando un nivel de significación del 0,000 para la $\chi^{2}$. Esta alternativa nos explicará sólo el 63,096 \% de la varianza, dato no obstante también aceptable. Las altas comunalidades nos aseguran la fiabilidad de las escalas. La obtención de cargas factoriales por encima del 0,4 \% aseguran la validez convergente. Finalmente, las alfa de Cronbach de 0,830 y 0,932 respectivamente para las dos dimensiones, en frente a una correlación de 0,461 entre estas dimensiones, asegura la validez discriminante

Dados estos análisis, y en aras a obtener unos resultados consistentes, este estudio va a observar en las modelizaciones estructurales causales posteriores la variable latente general que contrastamos en el AFC, y que llamaremos Resultado Total (RT), y la agregación de las dos medidas del modelo de AFE de 2 medidas, y que llamaremos Resultado Global (RG).

\subsection{Análisis de los Modelos Estructurales Causales, contraste de hipótesis}

Una vez validadas las escalas, la segunda fase en el uso del modelo de ecuaciones estructurales en dos fases se refiere a las relaciones de causalidad para contrastar la hipótesis considerada. En nuestro modelo nos planteamos el análisis de la influencia que tiene el desarrollo global de las TICs en la empresa en la explicación del desempeño empresarial. Dado ello, hemos atendido a las fases de especificación, identificación, estimación e interpretación.

El objeto de las fase de especificación es el traducir las hipótesis teóricas en un sistema de ecuaciones. El diagrama de pasos que recoge estas relaciones viene expuesto en la Figura 1. En esta fase vamos a considerar como variable latente exógena el desarrollo de las tecnologías de información y comunicación en la empresa $\left(\mathrm{TIC}=\xi_{1}\right)$, variable que estará explicada por los cuatro ítems que componen esta dimensión $\left(\mathrm{TIC}_{1}, \mathrm{TIC}_{2}\right.$, $\mathrm{TIC}_{3}$ y $\mathrm{TIC}_{4}$ ). En segundo lugar, la variables endógena relativa al desempeño la hemos medido nuevamente de dos formas: En primer lugar hemos observado el desempeño total $\left(\eta_{11}=\right.$ DT), como una variable agregada general de las 4 dimensiones contrastadas por el AFE de 4 factores y posteriormente por un AFC. En segundo lugar, nosotros también consideramos un modelos latente configurado por las dos dimensiones agregadas contrastadas por el AFE de 2 factores, y que denominamos Desempeño

Enc. Bibli: R. Eletr. Bibliotecon. Ci. Inf., Florianópolis, n. esp., 2º sem. 2008 
Global $\left(\eta_{12}=\mathrm{DG}\right)$. Esta variable endógena será explicada directamente por el desarrollo de las TIC en la empresa

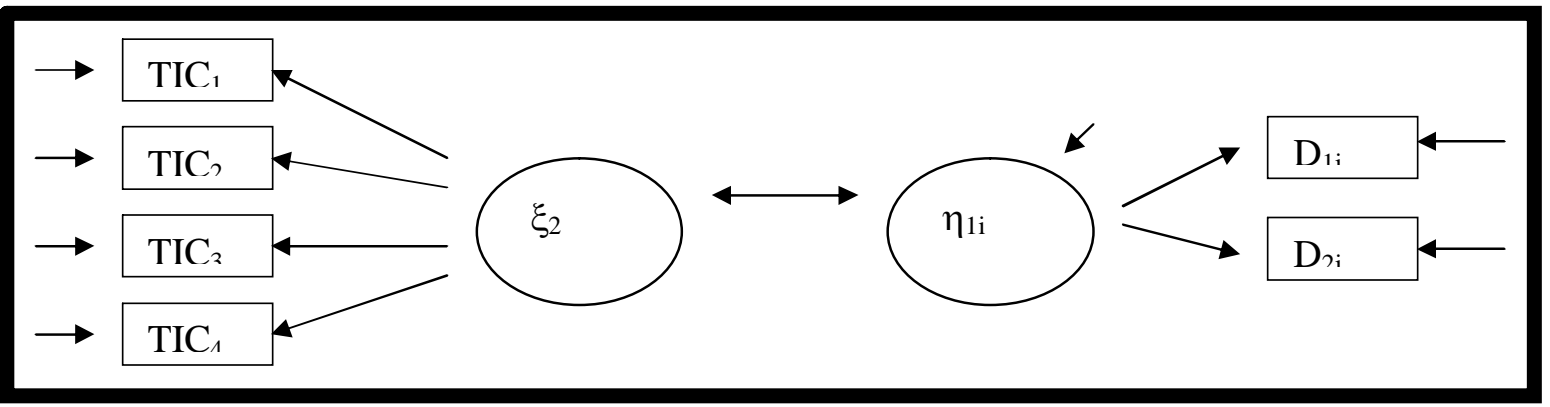

Figura 1. Diagrama de pasos de los modelos estructurales

La identificación implica que los parámetros del modelo puedan derivarse a partir de las varianzas y covarianzas entre las variables observables. Ambos modelos están sobre identificados, con grados de libertad positivos (Tabla 4).

El siguiente paso es estimar los resultados para buscar estimaciones sin sentido o teóricamente inconsistentes. Previamente a este análisis, debemos probar el ajuste del modelo, evaluado con medidas de ajuste absolutas, incrementales y de parsimonia. La mayoría de los indices alcanzan los valores deseados, reflejandu un ajuste aceptable del modelo (Tabla 4).

Tabla 4. Medidas de bondad de ajuste de los modelos estructurales

\begin{tabular}{|c|c|c|}
\hline & \multicolumn{2}{|c|}{ Modelos TIC-Desempeño } \\
\hline & TIC- DT & TIC-DG \\
\hline \multicolumn{3}{|l|}{ Identificación } \\
\hline G. libertad & 5 & 8 \\
\hline \multicolumn{3}{|l|}{ Estimación } \\
\hline \multicolumn{3}{|l|}{$\begin{array}{l}\text { M. absolutas de } \\
\text { ajuste }\end{array}$} \\
\hline Satorra-Bentler $\chi^{2}$ & 3,291 & 6,070 \\
\hline
\end{tabular}

El siguente paso es centrarse en el ajuste del modelo estructural estimado, para encontrar el nivel de significación de los parámetros estimados y la fiabilidad de las ecuaciones estructurales (Tabla 5). Podemos observar que los dos coeficientes son significativos y positivos. Además, la evaluación de las $\mathrm{R}^{2}$ nos señala que el desarrollo de las TIC en la empresa tiene una capacidad explicativa directa de los resultados del $10 \%$. Este hecho muy importante si tenemos en cuenta además todos los efectos indirectos inducidos por este desarrollo de las TIC. Este hecho corrobora nuestra teoría, confirmando la aceptación de nuestras hipótesis.

Enc. Bibli: R. Eletr. Bibliotecon. Ci. Inf., Florianópolis, n. esp., 2º sem. 2008 
Tabla 5. Parámetros estimados en los modelos estructurales

\begin{tabular}{|c|c|c|}
\hline & \multicolumn{2}{|c|}{ Modelos TIC- Desempeño } \\
\hline & TIC-DT & TIC- DG \\
\hline $\begin{array}{l}\text { Coeficiente } \beta_{3 \mathrm{i}} \text { en las ecuaciones } \eta_{1 \mathrm{i}}=\beta_{3 \mathrm{i}} \xi_{2}+ \\
\zeta_{3 \mathrm{i}} \\
\left(\mathrm{R}_{1 \mathrm{i}}=\beta_{3 \mathrm{i}} \mathrm{TI}+\zeta_{3 \mathrm{i}}\right)\end{array}$ & 0,298 & 0,326 \\
\hline $\begin{array}{l}\text { Significatividad estadística del coeficiente ( } t \\
>1,96)\end{array}$ & 4,087 & 3,308 \\
\hline Fiabilidad de las ecuaciones estructurales ( $\mathbf{R}^{2}$ ) & 0,089 & 0,106 \\
\hline
\end{tabular}

\section{CONCLUSIÓN}

El primer objetivo de este trabajo ha sido mostrar teóricamente la importancia de las Tecnologías de la Información y las Comunicaciones para las empresas, y su repercusión en la competitividad. El contraste empírico de las hipótesis nos ha revelado la importancia decisiva directa de las tecnologías de información y las comunicaciones en el devenir empresarial. Atendiendo a los planteamientos desarrollados en la parte teórica hemos corroborado la escala construida para medir el desarrollo de las TIC en la empresa. Esta investigación corrobora también la escala de medida de desempeño establecida. Verificados los constructos, nuestro estudio continúa con el contraste empírico del razonamiento teórico. El trabajo teórico remarca la importancia trascendental de las TIC en la explicación del desempeño empresarial y ello es también corroborado por nuestros datos. Dado ello, permitámonos enfatizar en algunas de las ventajas de las TIC de entre todas las explicadas en la sección teórica.

Las TIC pueden ayudar a las empresas a la reducción de costes tanto de entradaen nuevos negocios como en operaciones y transacciones. Ellas pueden favorecen la reducción del tiempo de lanzamiento al mercado de nuevos productos y a aprender a establecer rápidamente ofertas competitivas. Existen claras ventajas en constes en los modos en que las compañías operan en mercados virtuales, con lo que las teorías convencionales de aglomeración de empresas y competencia regional sufren modificaciones importantes.

Las nuevas innovaciones tecnológicas facilitan el proceso de comunicación y coordinación dentro de la empresa de las actividades de negocios. Las TIC incrementan la productividad fruto de los procesos de automatización que liberan a trabajadores de tareas rutinarias, y de la eliminación de costes de transacción. Además permiten la mayor capacitación de los empleados y el compartimiento de conocimiento e información de los mismos. Al mismo tiempo permiten plantear fórmulas creativas para mantener alta su satisfacción y motivación que a su vez contribuye a la calidad de los productos y a la imagen de la empresa.

Estas TIC permiten a la empresa estar al alcance de un gran número de gente de forma rápida y barata y sin límites geográficos. Ellas pueden permitir crear valor añadido a los clientes permitiendo la salida ágil al mercado tanto de nuevos productos y servicios, y la inclusión en catálogos web de estos con sus descripciones y precios para estar a mayor disposición de los clientes finales. Este hecho va aparejado además a un aumento de ingresos por el suministro de servicios adicionales debidos a la apertura de 
nuevos canales de distribución y venta. La interactividad de las nuevas tecnologías permite la posibilidad de crear productos y servicios innovadores y a medida de los clientes de un modo rápido, fácil y barato. Estos productos además pueden diferenciar a la empresa, contribuir a la fidelización del cliente, y evaluar la calidad de productos y servicios con la experiencia del consumidor, hecho que contribuye a la eficacia y eficiencia de los nuevos desarrollos.

Además facilita la comparación de precios, condiciones y características de los productos ofrecidos por proveedores, además de permitir incrementar las relaciones con proveedores, socios o colaboradores, que conducen a la creación de acuerdos de colaboración que facilitan el intercambio de información en tiempo real. Las nuevas tecnologías incrementan la habilidad de las empresas para cooperar a niveles diferentes de la cadena de suministro y facilita procesos de innovación complejos con relativo peso en interacciones intensivas proveedor-productor de servicio-usuario y sus correspondientes procesos de aprendizaje.

Las nuevas tecnologías apoyan la emergencia de comunidades virtuales que suprimen las barreras tradicionales entre empresas a lo largo de la cadena de valor. Ello puede implicar el compartimiento de los procesos de negocios entre grupos de empresas de diferentes sectores e industrias, hecho que puede desintegrar las estructuras de los canales de industrias tradicionales, abriéndose nuevos modos de comunicación e intercambio del conocimiento debido a nuevas formas de intercambio económico tales como los mercados electrónicos.

Además permiten un intercambio y el compartimiento de información entre empresas alejadas geográficamente, hecho que promueve el conocimiento y el aprendizaje externo. Las TICs incrementan la transparencia de procesos de negocios, a medida que diferentes empresas son capaces de comunicar e intercambiar conocimiento sin mucha dificultad a través de extranets y acceso remoto a bases de datos centrales. La expansión del conocimiento puede servir de alimento a procesos de cambio tecnológico y adopción a medida que las empresas conjugan diferentes combinaciones de individuos y recursos.

Uno de los factores fundamentales viene derivado de la potenciación del conocimiento. Las nuevas tecnologías extienden el proceso de integración y compartimiento de inteligencia entre empresas y negocios, permitiendo una planificación colaborativa, predicción y reemplazo de la gestión de la cadena de valor más allá de barreras nacionales y regionales. Dado ello, el mayor conocimiento de los gerentes de los cambios producidos por el nuevo entorno y las TICs pueden ser críticos enriqueciéndose mutuamente para enfrentarse al desarrollo de las nuevas tecnologías.

Podemos remarcar la importancia de nuestro estudio, tanto por la aplicación al sector hotelero español de todas las innovaciones que pueden ocasionar las TICs, las cuales han sido descritas en el apartado teórico, como también por la confirmación de la bondad los modelos planteados y la corroboración empírica de su aplicación en el sector estudiado. Es más, si además contemplamos la utilidad práctico-empresarial de nuestro estudio, nuestro análisis remarca claramente qué un mayor desarrollo de las TICs en las empresas suelen conducir a un mejor desempeño empresarial. Es claro que las empresas necesitan mejorar su conocimiento del entorno competitivo que abre el desarrollo de Internet de modo que los directivos puedan elegir las mejores estrategias, y por tanto se beneficien de las relaciones entre las ITCs y la mejora del desempeño.

Enc. Bibli: R. Eletr. Bibliotecon. Ci. Inf., Florianópolis, n. esp., 2º sem. 2008 
Dado ello, planteamos la necesidad por parte de las empresas de utilizar y desarrollar de forma intensiva las TICs en las formas explicadas.

Las inferencias de este estudio, sin embargo, deberían ser subordinadas por las posibles limitaciones de la muestra y las metodologías utilizadas. Consideramos finalmente relevante la necesidad de trabajos futuros que enriquezcan nuestro análisis. Futura investigación podría utilizar las mismas perspectivas con diferentes muestras tanto en el sector turístico como en otros varios, o incluso podría utilizar diferentes metodologías.

\section{REFERENCIAS}

ALTINAY, L., WANG, C.L., 2006. The role of prior knowledge in international franchise partner recruitment. International Journal of Service Industry Management, 17 (5), 430-443.

ANDERSON, J.C., GERBING, D.W., 1988. Structural equation modeling in practice: a review and recommend two step approach. Psychological Bulletin, 103 (3), 453-460. BARNATT, C., 1996. Management Strategy and Information Technology. Text and Readings. International Thomson Business Press, London.

BARTON, P.S., PETERS, D.H., 1993. The ASB Bank: An IT case study in sustained competitive advantage. Journal of Strategic Information System, 1, 165-170.

BASTAKIS, C., BUHALIS, D., BUTLER, R., 2004. The perception of small and medium sized tourism accommodation providers on the impacts of the tour operators' power in Eastern Mediterranean. Tourism Management, 25, 151-170.

BERGERON, F., BLUTEAU, Ch., TAYMOND, L., 1991. Identification of Strategic Information Systems Opportunities: Applying and comparing two methodologies. MIS Quarterly, 15, 89-101.

BHARADWAJ, A. S., 2000. A resource-based perspective on information technology capability and firm performance: An empirical investigation. MIS Quarterly, 24 (1), 169-196.

BOLLEN, K.A., 1989. Structural Equations with Latent Variables. Wiley, New York.

BOOTH, M., PHILIP, G., 1997. Technology, competencies and competitiveness: the case for reconfigurable and flexible strategies. Journal of Business Research, 41, 2940.

BREY, E.T., SO., S.I., KIM, D.Y. MORRISON, A.M., 2007. Web-based permission marketing: Segmentation for the lodging industry. Tourism Management, 28, 14081416.

BROADBENT, M., WEILL, P., ST. CLAIR, D., 1999. The implications of information technology infrastructure for business process redesign. MIS Quarterly, 23 (2), 159-182.

BRUQUE, S., VARGAS, A., HERNÁNDEZ, M.J., 2003. Determinantes del valor competitivo de las tecnologías de la información. Una aplicación al sector de distribución farmacéutica. Revista Europea de Dirección y Economía de la Empresa, 12 (4), 101-124.

BUHALIS, D., 1998. Strategic use of information technologies in the tourism industry. Tourism Management, 19 (5), 409-421. 
BUHALIS, D., LICATA, M.C., 2002. The future of E-tourism intermediaries. Tourism Management, 23(2), 207-220.

CAMISON, C., 1999. La medición de los resultados empresariales desde una óptica estratégica: construcción de un instrumento a partir de un estudio Delphi y aplicación a la empresa industrial española en el periodo 1983-96. Revista de contabilidad y Tributación, 62, 201-264.

CAMISON, C., 2000. Strategic attitudes and information technologies in the hospitality business: and empirical analysis. International Journal of Hospitality Management, 19 (2), 125-143.

CAMPBELL-HUNT, C., 2000. What have we learned about generic competitive strategy? A Meta-Analysis. Strategic Management Journal, 21, 127-154.

CHAPELET, B., TOVSTIGA, G., 1998. Development of a research methodology for assessing a firm's business process-related technologies. International Journal Technology Management, 15 (1/2), 10-13.

CHEN, Y., ZHU, J., 2004. Measuring Information Technology's Indirect Impact on firm performance. Information Technology and Management, 5, 9-22.

CHIOU, W.B., WANG, C.S., LEE, H.Y., 2008. Virtual experience vs brochures in the advertisement of scenic spots: How cognitive preferences and orde effects influence advertising effects on consumers. Tourism Management, 29, 146-150.

CLEMONS, E.K., ROW, M.C., 1991. Sustaining IT advantage. The role of structural differences. MIS Quarterly 275-292.

CONESA, P., 2003. Alcance y Validación de la Política de Promoción Europea de la Sociedad de la Información a Través del Programa Ten-Telecom en la Aplicación de una Plataforma Multisectorial de Comercio Electrónico en tres Países de la Unión Europea: España, Francia y Grecia. Doctoral Thesis. Universidad Politécnica de Valencia. Departamento de Organización de Empresas, Economía Financiera y Contabilidad.

COOPER, C., FLETCHER, J., GILBERT, D., WANHILL, S., 1999. Tourism Principles and Practice (second edition). Addison Wesley Longman, Harlow.

COULTER, M.K., 1997. Strategic Management in Action. Prentice-Hall, Upper Sanddle River, New Jersey.

CHATTOPADHYAY, P., GLICK, W., MILleR, C.C., HUBER, G., 1999. Determinants of executive beliefs: Comparing functional conditioning and social influence. Strategic Management Journal, 20 (8), 763-789.

DARR, W., ARGOTE, L., EPPLE, D., 1995. The acquisition, transfer and depreciation of knowledge in service organizations: Productivity in franchises. Management Science, 41, 1750-1762.

DEV, C.S., OLSEN, M.D., 2000. Marketing challenges for the next decade. Cornell Hotel and Restaurant Administration Quarterly, 41 (1), 41-47.

DEVARAJ, S., KOHLI, R., 2003. Performance Impacts of Information Technology: Is Actual Usage the Missing Link? Management Science, 49 (3), 273-289.

DRURY, D., 1997. The dialectic of IT chargeback systems. International Journal Technology Management, 14 (5), 496-512.

DUSSAUGE, P., HART, S., RAMANANTSOA B., 1994. Strategic Technology Management. John Wiley \& Sons, Chichester. 
ENG, T.Y., 2004. Implications of the Internet for knowledge creation and dissemination in clusters of Hi-tech Firms. European Management Journal, 22 (1), 87-98

FAYOS-SOLA, E., PEDRO BUENO, A., 2001. Globalization, national tourism policy and international organizations. In: WAHAB, S., COOPER, C. (Eds.) Tourism in the Age of Globalisation. Routledge, London, p. 45-65.

FEIERTAG, H., 2007. Use today's technology to plan meetings. Hotel and Motel Management. Septiembre, 3.

FORNELL, Cl., ROBINSON, W.T., WERNERFELT, B., 1985. Consumptive experience and sales promotion expenditure. Management Science, 31, 1084-1085.

GARRIGÓS, F., NARANGAJAVANA, Y., 2002. El proceso de globalización de la industria hotelera española: Una visión retrospectiva de las cadenas españolas en la década de los noventa. Estudios Turísticos, 152, 35-64.

GARRIGÓS, F., NARANGAJAVANA, Y., 2006. Capacidades Directivas y Nuevas Tecnologías en el Sector Turístico. Editorial Atenea. Castellón, España.

GARRIGÓS, F., 2008. Interrelationships between professional virtual communities and social networks, and the importance of virtual communities in creating and sharing knoweldge. CAMISÓN, César et al., (ed.). Handbook of Research on Knowledge Management in Virtual Organizations and Networks. Idea Group, INC. En prensa HAIR, J.F., ANDERSON, R.E., TATHAM, R.L., BLACK, W.C., 1998. Multivariante Date Análisis. Spanish Edition: Análisis Multivariante (5 ${ }^{\text {th }}$ ed.), 1999. Prentice Hall International, Madrid.

HAMEL, G., 1991. Competition for competence and inter-partner learning withing internantional strategic alliances. Strategic Management Journal, 12, 83-103.

HANSON, D., DOWLING, P., HITT, M.A., IRELAND, R.D., HOSKISSON, R.E., 2002. Strategy Management, Competitiveness \& Globalisation. Pacific Rim Edition. Nelson, Southbank Victoria.

HARVEY, M., GRIFFITH, D., NOVICEVIC, M., 2000. Development of 'Timescapes' to effectively manage global inter-organizational relational communications. European Management Journal, 18 (6), 646-661.

HJALAGER, A., 2006. Stages in the economic globalization of tourism. Annals of Tourism Research, 34 (2), 437-457.

HOLJEVAC, I. A., 2003. A vision of tourism and the hotel industry in the $21 \mathrm{st}$ century. Hospitality Management, 22, 129-134.

HOLLAND, C., LOCKETT, G., BLACKMAN, I., 1992. Planning for electronic data interchange. Strategic Management Journal, 13 (7), 539-550.

INGRAM, P., BAUM, J., 1997. Chain affiliation and the Manhattan hotels, 1898-1982. Administrative Science Quarterly, 42, 68-102.

KIM,B., BARUA, A., WHINSTON, A.B., 2002. Virtual field experiments for a digital economy: A new research methodology for exploring an information economy. Decision Support Systems, 32 (3), 215-231.

LAUDON, K.C., LAUDON, J.P., 1998. Management Information Systems. New Approaches to Organization and Technology $\left(5^{\text {th }}\right.$ ed). Prentice Hall International Inc, London. 
LOEBBECKE, C., WAREHAM, J., 2003. The impact of E-Business and the Information Society on 'strategy' and 'strategic planning': An assessment of new concepts and challenges. Information Technology and Management, 4, 165-182.

LYON, D.W., LUMPKIN, G.T., DESS, G.G., 2000. Enhancing entrepreneurial orientation research: Operationalizing and measuring a key strategic decision making process. Journal of Management, 26 (5), 1055-1085.

MAHMOOD, M.A., MANN, G.J., 1993. Measuring the organizational impact of information technology investment: An exploratory study. Journal of Management Information Systems, 10 (1), 97-122.

MATA, F.J., FUERST, W.L., BARNEY, J. B., 1995. Information technology and sustained competitive advantage: A resource-based analysis. MIS Quarterly, 19 (4), 487-506.

MINTZBERG, H., AHLSTRAND, B., LAMPEL, J., 1998. Strategy Safary. A Guided Tour Through the Wilds of Strategic Management. Prentice Hall, London.

PFEFFER, J., 1992. Managing with Power: Politics and Influence in organizations. Harverd Business School Press, Boston.

PIZAM, A., 1999. Life and tourism in the year 2050. International Journal of Hospitality Management, 18 (4), 331-343.

O' CONNOR, P., FREW, A.J., 2004. An evaluation methodology for hotel electronic channels of distribution. International Journal of Hospitality Management, 23 (2), 179-199.

PIZAM, A., MANSFELD, Y., 1999. Consumer Behaviour in Travel \& Tourism. The Haworth Hospitality Press, New York.

PORTER, M.E., 1980. Competitive Strategy: Techniques for Analyzing Industries and Competitors. The Free Press.

POWELL, T.C., DENT-MICALLEF, A., 1997. Information technology as competitive advantage: The role of human, business, and technology. Strategic Management Journal, 18 (5), 375-405.

QUIRCHMAYR, G., SCHIMK, R., 1994. TIS/MM-PORTUGAL. In: SCHERTLER, W., SUMID, B., TJOA, A.M., WERTHNER, H. (Eds.), Information and Communication Technologies in Tourism. Springer-Verlag Wien, New York.

REGNÉR, P., 2003. Strategy creation in the periphery: Industive versus deductive strategy making. Journal of Management Studies, 40(1), 57-82.

RUSS, M., CAMP, S., 1997. Strategic alliances and technology transfer. an extended paradigm. International Journal Technology Management, 14 (5), 513-527.

RUST, R.T., ZAHORIK, A.K., KEININGHAM, T.L., 1996. Service Marketing. Harper Collins, New York.

SAMBAMURTHY, V., BHARADWAJ, A., GROVER, V., 2003, Shaping agility through digital options: Reconceptualizing the role of information technology in contemporary firms. MIS Quarterly, 27 (2), 237-263.

SANTHANAM, R., HARTONO, E., 2003. Issues in Linking Information Technology Capability to Firm Performance. MIS Quarterly, 27 (1), 125-153.

SATORRA, A., BENTLER, P.M., 2001. A scaled difference Chi-Square test statistic for moment structure analysis. Psychometrika, 66 (4), 507-514.

SCHOEMAKER, P.J.H., 1997. Disciplined imagination. From scenarios to strategic options. International Studies of Marketing and Organizations, 27(2), 43-70. 
SEIDERS, K., BERRY, L.L., GRESHAM, L.G., 2000. Attention, retailers! How convenient is your convenience strategy? Sloan Management Review, 41 (3), 79-89. SHARMA, A., 1997. Professional as agent: Knowledge asymmetry in agency exchange. Academy of Management Review, 22(3), 229-236.

SINHA, I., 2000. Cost transparency: The Net's real threat to prices and brands. Harvard Business Review, 18 (2), 43-50.

SLATER, S. F., OLSON, E.M., 2000. Strategy type and performance: The influence of sales force management. Strategic Management Journal, 21, 813-829.

STAMBOULIS, Y., SKAYANNIS, P., 2003. Innovation strategies and technology for experience-based tourism. Tourism Management, 24, 35-43.

STOKES BERRY, F., 1998. Explain managerial acceptance of expert systems. Public Productivity and Management Review, 20 (3), 323-335.

SZEINBACH, SH. L., BARNES, J. H., GARNER, D.D., 1997. Use of Pharmaceutical Manufacturers' value-added services to build customer loyalty. Journal of Business Research, 40, 229-236.

TANRIVERDI, H., 2005. Information technology relatedness, knowledge management capability, and performance of multibusiness firms. MIS Quarterly, 29 (2), 311-334.

TEIGLAND, R., WASKO, M.M., 2003. Integrating knowledge through information trading: Examining the relationship between boundary spanning communication and individual performance. Decision Sciences, 34 (2), 261-286.

TIPPINS, M.J., SOHI, R. S., 2003. IT competency and firm performance: Is organizational learning a missing link?. Strategic Management Journal, 24 (8), 745761

VANHOVE, N., 2001. Globalisation of tourism demand, global distribution systems and marketing. In: WAHAB, Salah, COOPER, Chris (Eds). Tourism in the Age of Globalisation. Routledge, London. p.123-155.

VENKATRAMAN, N., RAMANUJAM, V., 1986. Measurement of business performance in strategic research: A comparison approach. Academy of Management Review, 11 (4), 801-814.

WADE, M., HULLAND, J., 2004. Review: The Resource-Based view and information systems research: Review, extension, and suggestions for future research. MIS Quarterly, 28 (1), 107-142.

WAHAB, S., COOPER, C., 2001. Tourism in the Age of Globalisation. Routledge, London.

WALTERS, B.A., JIANG, J., KLEIN, G., 2003. Strategic information and strategic decision making: The EIS/CEO interface in smaller manufacturing companies.

Information \& Management, 40, 487-495.

WATKINS, E., 2000. Building the perfect site. Lodging Hospitality, 56 (3), 38-40.

WEILL, P., ROSS, J., 2005. A matrixed approach to designing IT governance. MIT Sloan Management Review, 46 (2), 26-34.

WILSON, R., 2007. Internet hotel reservations. The "terms and conditions" trap. Cornell Hotel and Restaurant Administration Quarterly, 48 (4), 361-369

WÖBER, K.W., 2003. Information supply in tourism management by marketing decision support systems. Tourism Management, 24, 241-255. 
YOUNDT, M.A., SUBRAMANIAM, M., SNELL, S.A., 2004. Intellectual capital profiles: An examination of investment $s$ and returns. Journal of Management Studies, 41 (2), 335-361.

\begin{abstract}
The introduction of Information and Communication Technologies has brought about a change in company behaviour. This change has proved to be of particular importance in the tourism sector, due to its own peculiarities. This work examines how the development of new technologies has affected the way in which tourism enterprises compete and develop their tasks. In addition our work tests empirically the impact that the development of information technologies inside the enterprise has on the explanation of firm performance. In order to achieve this aim we use a questionnaire addressed to hospitality managers, and Structural Equations Modelling methodology. The study highlights the benefits associated with the use of IT, together with possible formulae to improve the managerial task.
\end{abstract}

Key words: Information and Communication Technologies; Structural Equations; Hospitality Sector.

Originais recebidos em: 30/09/2008

Texto aprovado em: 06/11/2008 